\title{
Full-Vector Signal Acquisition and Information Fusion for the Fault Prediction
}

\author{
Lei Chen, ${ }^{1,2}$ Jie Han, ${ }^{1}$ Wenping Lei, ${ }^{1}$ Yongxiang Cui, ${ }^{1}$ and Zhenhong Guan ${ }^{1}$ \\ ${ }^{1}$ Institute of Vibration Engineering, Zhengzhou University, Zhengzhou 450001, China \\ ${ }^{2}$ School of Chemical Engineering and Energy, Zhengzhou University, Zhengzhou 450001, China
}

Correspondence should be addressed to Lei Chen; chenlei@zzu.edu.cn

Received 21 September 2015; Revised 6 January 2016; Accepted 28 February 2016

Academic Editor: Robert C. Hendricks

Copyright (C) 2016 Lei Chen et al. This is an open access article distributed under the Creative Commons Attribution License, which permits unrestricted use, distribution, and reproduction in any medium, provided the original work is properly cited.

Fault prediction is the key technology of the predictive maintenance. Currently, researches on fault prediction are mainly focused on the evaluation of the intensities of the failure and the remaining life of the machine. There is lack of methods on the prediction of fault locations and fault characters. To satisfy the requirement of the prediction of the fault characters, the data acquisition and fusion strategies were studied. Firstly, the traditional vibration measurement mechanism and its disadvantages were presented. Then, the full-vector data acquisition and fusion model were proposed. After that, the sampling procedure and information fusion algorithm were analyzed. At last, the fault prediction method based on full-vector spectrum was proposed. The methodology is that of Dr. Bently and Dr. Muszynska. On the basis of this methodology, the application study has been carried out. The uncertainty of the spectrum structure can be eliminated by the designed data acquisition and fusion method. The reliability of the diagnosis on fault character was improved. The study on full-vector data acquisition system laid the technical foundation for the prediction and diagnosis research of the fault characters.

\section{Introduction}

As the key technology of the predictive maintenance [1], fault prediction is utilized to predict the operating condition of the machine and give an early warning of the failure which may occur in the future. Researchers have conducted lots of work on the aspects of machine fault diagnosis [2-6], operation status prediction [7-10], and feature extraction and information fusion [11-13], and the application of fault prediction has been proved to be favorable to instruct production dispatch and equipment maintenance. However, existing researches on mechanical fault prediction mainly focus on the forecasting of single specific fault trend and evaluating equipment's residual life. There are few studies and reports about the aspect of fault character, failure type, and failure position $[14,15]$.

To analyze the fault source and predict the fault character, failure type, and failure position of the equipment, one of the feasible means is to predict the trend of frequency spectrum. The failure type which may occur can be determined by analyzing the predicted spectrum structure $[16,17]$. To ensure the reliability of the prediction, the acquired signal should more faithfully and comprehensively reflect the operation status of the machine. At the same interface of rotating machine, the signal spectrum of different vibration will be obtained because of the different installation direction of the transducers. It is obvious that the information collected by a single transducer is not comprehensive [16]. Including the Chinese Power Utility Industry, industry wide of a single transducer to assess dynamics has been replaced by use of two or more transducers and key-phasor as fully described in detail in the classic works by Dr. Bently et al. [18]. Dr. Bently's classic work methodically and accurately explains in great detail the use of diagnostic equipment, theory, and applications. On the basis of these methodologies, the application has been carried out in this study. At the same test position, spectrum structure with one installation direction may be different from that of another direction. An error diagnosis may be made by analyzing such a signal spectrum. In order to make a comprehensive prediction and feasible diagnosis, making information fusion becomes necessary. The fusion signal should more faithfully and comprehensively 


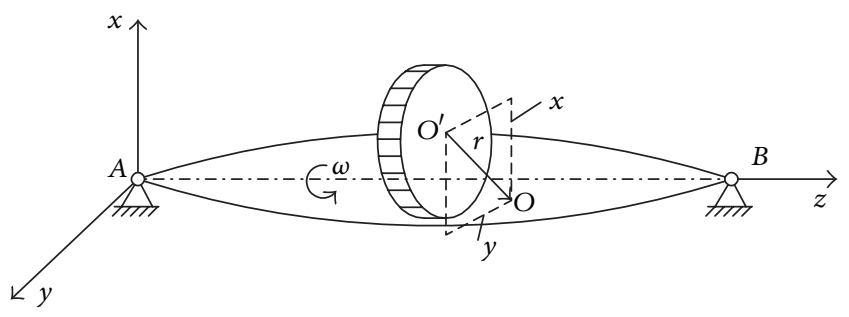

Figure 1: Jeffcott rotor.
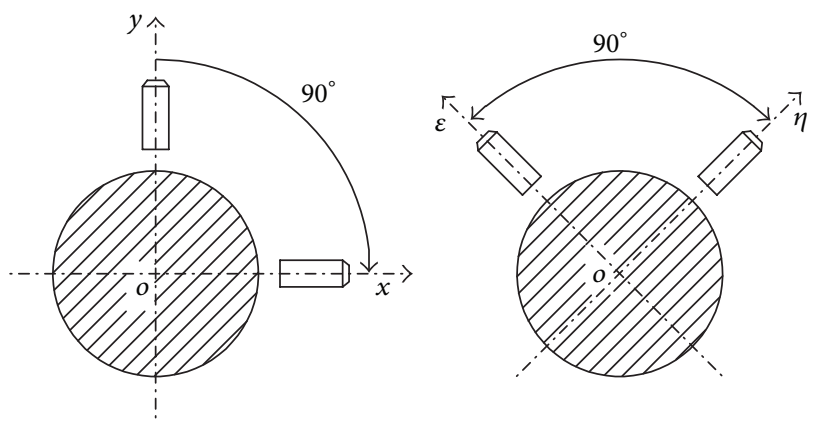

FIGURE 2: Layout of transducers.

reflect the machine operating condition. The fusion signal's spectrum is not concerned with the installation direction of the transducer. The failure type can be predicted by analyzing such a signal's spectrum trend. Therefore, this paper will take the rotating machine as the research object. Firstly, the data acquisition and signal fusion model for the fault character prediction will be analyzed. Then, the vibration signal acquisition system based on full-vector spectrum will be designed. Lastly, the signal fusion algorithm will be further studied.

\section{Vibration Mechanism and Signal Monitoring}

Generally, the rotating machine has complex structure. For the convenience of analysis, the mechanical model of the machine rotor was simplified as a disc with mass $m$ and an elastic shaft with no mass, and the shaft ends are supported by a rigid bearing and a bearing base [19-21], as shown in Figure 1.

Assume that the two support points of the rotor are in the same horizontal line and the disc is located in the center of the two fulcrums of the rotor. When the rotor is rotating, it generates dynamic torsion with diameter $r$ due to the centrifugal inertia force. There are two kinds of rotations at this time: (1) rotation of the disc around the axis $A O^{\prime} B$ and (2) rotation of the plane of the $A O^{\prime} B$ and the $A O B$ of the bearing line around the $A B$ axis $[16,22]$.

Assuming the mass of the disc is $m$, the elastic recovery force of the rotor is $F$ :

$$
F=-k r
$$

where $k$ is the rotor stiffness coefficient, $r=O O^{\prime}$. The differential equation of motion of the disc is

$$
\begin{aligned}
& m \ddot{x}=F_{x}=-k x, \\
& m \ddot{y}=F_{y}=-k y,
\end{aligned}
$$

Make $\omega^{2}=\frac{k}{m}$,

$$
\begin{aligned}
& \ddot{x}+\omega^{2} x=0, \\
& \ddot{y}+\omega^{2} y=0 .
\end{aligned}
$$

Its solution can be written as

$$
\begin{aligned}
& x=X \cos \left(\omega t+\varphi_{x}\right) \\
& y=Y \cos \left(\omega t+\varphi_{y}\right),
\end{aligned}
$$

where $X$ stands for the amplitude of the motion of the disc center in the direction of $x$ and $Y$ stands for the amplitude of the motion of the disc center in the direction of $y \cdot \varphi_{x}$ and $\varphi_{y}$ stand for the phase angles of the corresponding direction and $t$ stands for the time. The rotor vibration of $x$ - and $y$ directions of a stationary rotating machinery is a combination of motion orbits for some harmonic waves $w_{i}(i=1,2, \ldots)$.

Generally, the vibration of the rotating machine is measured by noncontact eddy current transducers. The transducers are installed with the $X-Y$ layout or $V$ installation as is shown in Figure 2. The measured vibration signal of $x$ or $y$ transducer is a combination of motion orbits for some harmonic waves $w_{i}(i=1,2, \ldots)$. For a certain harmonic $i$, suppose

$$
\begin{aligned}
X_{c i} & =X_{i} \cos \emptyset_{x i}, \\
X_{s i} & =X_{i} \sin \emptyset_{x i}, \\
Y_{c i} & =Y_{i} \cos \emptyset_{y i}, \\
Y_{s i} & =Y_{i} \sin \emptyset_{y i},
\end{aligned}
$$

where $X_{i}$ stands for the amplitude of vibration signal of the harmonic $i$ in the direction $x ; Y_{i}$ stands for the amplitude of vibration signal of the harmonic $i$ in the direction $y ; \emptyset_{x i}$ stands for the phase angle of the harmonic $i$ in the direction $x ; \emptyset_{y i}$ stands for the phase angle of the harmonic $i$ in the direction $y ; X_{c i}$ stands for the amplitude of vibration in the direction $x$ of harmonic $i$ when $t=0 ; Y_{c i}$ stands for the amplitude of vibration in the direction $y$ of harmonic $i$ when $t=0 ; X_{s i}$ and $Y_{s i}$ have no physical meaning and were used for the geometry calculation of harmonic track. 


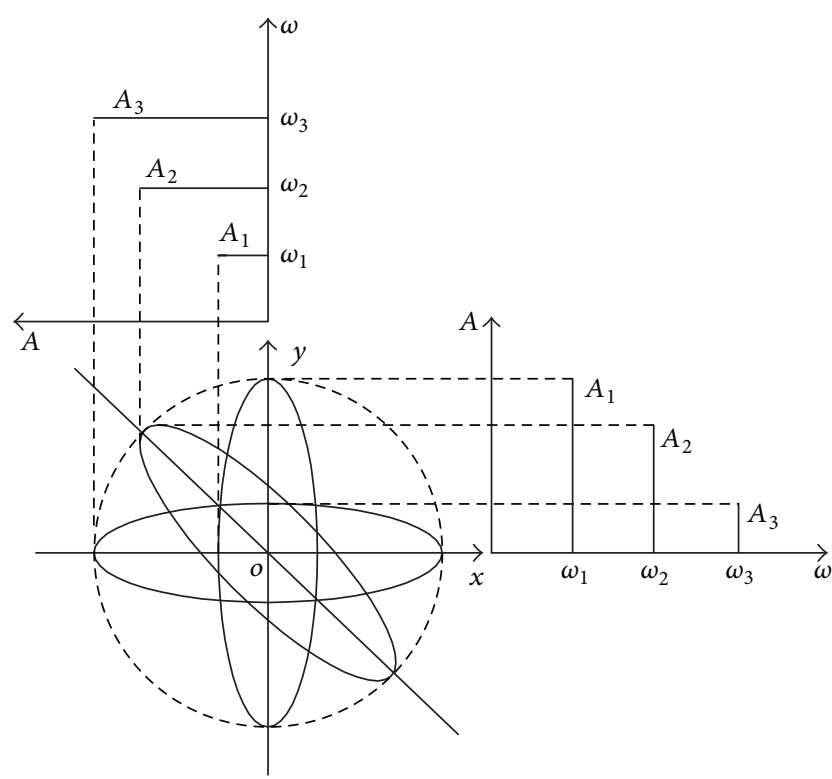

FIGURE 3: Spectrum structure error of transducers in different directions.

Movement equation of $x$ - and $y$-direction can be expressed as

$$
\begin{aligned}
x & =\sum_{i=1}^{\infty} X_{i} \cos \left(w_{i} t+\emptyset_{x i}\right)=\operatorname{Re}\left(\sum_{i=1}^{\infty} \bar{X}_{i} e^{j w_{i} t}\right) \\
& =\operatorname{Re}\left[\sum_{i=1}^{\infty}\left(x_{c i}+j x_{s i}\right) e^{j w_{i} t}\right], \\
y & =\sum_{i=1}^{\infty} Y_{i} \cos \left(w_{i} t+\emptyset_{y i}\right)=\operatorname{Re}\left(\sum_{i=1}^{\infty} \bar{Y}_{i} e^{j w_{i} t}\right) \\
& =\operatorname{Re}\left[\sum_{i=1}^{\infty}\left(y_{c i}+j y_{s i}\right) e^{j w_{i} t}\right] .
\end{aligned}
$$

In the above formula, $\bar{X}_{i}$ and $\bar{Y}_{i}$ represent the complex amplitudes of the vibration signals in the directions $x, y$ of the harmonic $i(i=1,2,3, \ldots)$; the trajectories of each harmonic are an ellipse; that is,

$$
\begin{aligned}
\left(Y_{c i}^{2}\right. & \left.+Y_{s i}^{2}\right) x_{i}^{2}+\left(X_{c i}^{2}+X_{s i}^{2}\right) y_{i}^{2} \\
& -2\left(X_{c i} Y_{s i}+X_{s i} Y_{c i}\right) x_{i} y_{i}=\left(X_{s i} Y_{s i}-X_{c i} Y_{c i}\right)^{2} .
\end{aligned}
$$

Suppose there exists a vibration signal with three harmonics. The angular frequencies of the harmonics are $w_{3}$, $w_{2}$, and $w_{1}$. The harmonics have the same length of the major axis $A$. The angles between the elliptical major axis and the $x$-direction are $\pi / 2,3 \pi / 4$, and 0 , as shown in Figure 3 . Figure 3 indicates that when measuring the same signal with transducer in different directions, different frequency spectrums will be obtained. That is to say, whether the transducer mounts in $x$-direction or in $y$-direction, the acquired signal in one signal transducer is one-sided for fault diagnosis. The reliability of conclusion concerning fault diagnosis and fault character prediction with such signals cannot be guaranteed. In order to obtain the reliable fault diagnosis and prediction, it is required to overcome the onesidedness of the vibration information obtained by a single transducer. One-sided information can be overcome by keyphasor [18]. Information fusion of the transducers in data layer is a feasible mean. In this study, full-vector signal is fused in the process of vibration data acquisition, and the full-vector spectrum will be extracted for the purpose of fault diagnosis and prediction.

\section{Full-Vector Data Acquisition and Information Fusion}

3.1. Full-Vector Data Acquisition. As is shown in Figure 4, transducers of $x$ - and $y$-direction sampled integer periodic vibration signal synchronously. The vibration acquired by the transducers of $x$ - and $y$-direction is fused based on fullvector spectrum in the data preprocessing stage, and a set of full-vector signals is obtained [18]. In order to obtain the fullvector phase spectrum of the vibration, a key phase trigger signal is needed. A key phase transducer is used to provide reference position of vibration phase and measure rotational speed. The data sampling process is controlled by a fieldprogrammable gate array (FPGA). Application running on the central processing unit (CPU) completes the full-vector fusion operation and obtains feature vectors for predicting and diagnosing.

3.2. Control Logic. The sampling process was controlled by a FPGA. The control logic is shown in Figure 5.

FPGA receives sampling parameters and instructions such as sampling frequency, sampling length, and sampling start from the CPU and converts them into the sampling control logic of analog-to-digital (AD) converter. As for the rotating machine, due to the importance of speed and phase for the fault diagnosis, the sampling is triggered by an external key. When the falling edge of the trigger signal comes, integrated periodic signal was acquired synchronously [23]. As is shown in Figure 6, the key phase transducer generates a level jump in every turn of the rotor. The interval between the two jumps is the rotation period. After the sampling instruction is issued, current speed is calculated in the FPGA and sampling rate is set according to the speed. AD conversion starts when the first pulse falling edge comes after setting the sampling parameters. Continuous $2^{N}$ sampling points are acquired for each channel. The signals of $x$ - and $y$-channel are acquired synchronously. Each sampling signal contains $W$ cycles and each cycle contains $2^{M}$ sampling points. Then, the sampling length $2^{N}=W \times 2^{M}$. The synthetic signal of $X$ and $Y$ sensor is a motion ellipse. The major axis of the ellipse, called main vector of the full-vector spectrum, reflects the most significant vibration. No matter where the transducer mounts, the main vibration vector is the maximum amplitude of the vibration in the motion plane. The vibration signal can be considered as superposition of a series of harmonic signals. The main vibration vectors of each harmonic can be obtained 


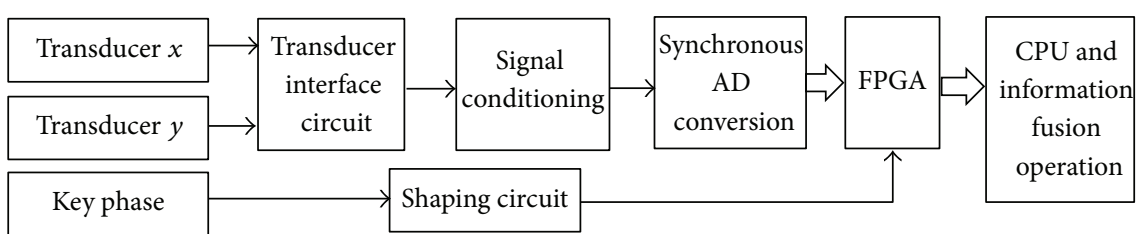

FIGURE 4: Structure of full-vector data acquisition.

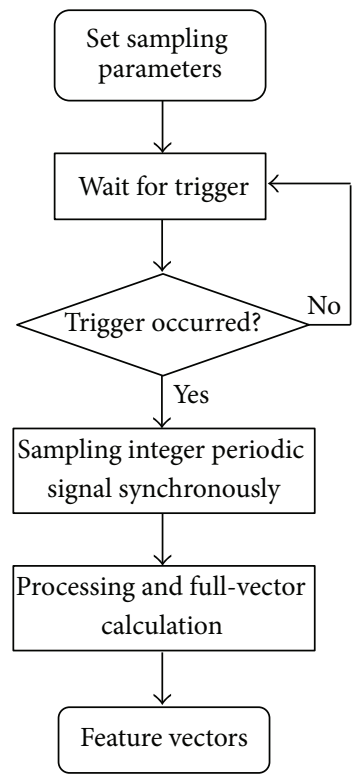

FIGURE 5: Procedure of full-vector acquisition.

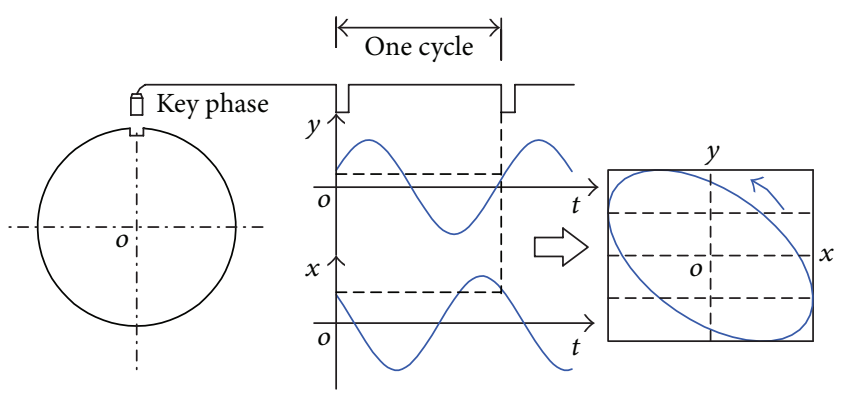

FIGURE 6: Diagram of synchronous integrated period sampling.

by data fusion, and then the full-vector spectrum is obtained. The obtained full spectrum is good for fault diagnosis and prediction.

3.3. Fusion Algorithm. According to the theory of full-vector spectrum, the main vector of vibration harmonic can be simplified as a simple calculation by FFT. Assume that $x_{i}$ and $y_{i}$ are the signals acquired by the transducers $x$ and $y$. Here, $i=0,1,2, \ldots, N-1$, where $N$ is the sampling points. A complex sequence $z_{i}$ can be constituted by $x_{i}$ and $y_{i}$; that is, $z_{i}=x_{i}+j y_{i} . Z_{k}$ can be obtained by Fourier transform of $z_{i}$ data series; here, $k=1,2, \ldots, N / 2-1$. After a series of calculations, (7) can be obtained. Hence,

$$
\begin{aligned}
& R_{L k}=\frac{1}{2 N}\left[\left|Z_{k}\right|+\left|Z_{N-k}\right|\right] \\
& R_{S k}=\frac{1}{2 N}\left[\left|Z_{k}\right|-\left|Z_{N-k}\right|\right] \\
& \qquad\left(k=0,1,2, \ldots, \frac{N}{2}-1\right),
\end{aligned}
$$

where $R_{L k}$ and $R_{S k}$ are the main and assistant vibration vectors. Since the synthetic signal of $X$ and $Y$ transducer at each harmonic is a motion ellipse, the major axis of the ellipse reflects the most significant vibration. $R_{L k}$ is half major axis of the ellipse at the frequency point $k$. $R_{S k}$ is half minor axis of the ellipse at the frequency point $k . R_{L k}$ is the maximum amplitude of the vibration in the motion plane. On the same rotor section, $R_{L k}$ and $R_{S k}$ are not affected by the radial mounting position of the two transducers installed vertically with each other. The full-vector spectrum, spectrum of $R_{L k}$, is the synthesis of two homologous transducers' information. Its spectral structure can reflect the operation state of the machine comprehensively. Fusion calculation in the data acquisition system can be carried out according to Figure 7 .

\section{Experiment and Discussion}

4.1. Experiment. To verify the correctness of the data acquisition system and fusion theory, an online monitoring system was installed in a thermal power plant. The Bently Nevada $3300 X L$ series of sensor systems were used in the system. And the eM3000 series data acquisition system was provided by Zhengzhou Expert P.D.E. Co., Ltd. Arrangement of measuring points on the feed water pump of steam turbine is shown in Figure 8. Two eddy current transducers are installed with $V$ layout at the position of input shaft bearing of the turbine. Vibration signals were acquired by synchronous integrated period sampling. The sampling frequency is $2000 \mathrm{Sa} / \mathrm{s}$, sampling length is 1024 points, and the rotor speed is $2930 \mathrm{r} / \mathrm{min}$. The signals of $x$ - and $y$-direction of the same cross section of an axis of the feed pump for a turbine are shown in Figure 9. The amplitude of first-order frequency, the amplitude of second-order frequency, and possible conclusions are shown in Table 1. Although the signal vibration amplitudes are all within the normal limits, there are obvious differences between the $x$ and $y$ signals in both time and frequency domain. According to the signal of $x$-direction, it seems that the vibration amplitude mainly relates to the double frequency, and perhaps there exists fault of misalignment. 
TABLE 1: Difference of the $x$-direction and $y$-direction and fusion signals.

\begin{tabular}{lccc}
\hline Signal & Amp. of 1st order $(\mu \mathrm{m})$ & Amp. of 2nd order $(\mu \mathrm{m})$ & Possible conclusions \\
\hline$x$-direction & 2.61 & 3.19 & Fault with double frequency \\
$y$-direction & 5.42 & 2.41 & Fault with rotating frequency \\
Fusion signal & 5.52 & 3.97 & Fault with rotating frequency \\
\hline
\end{tabular}

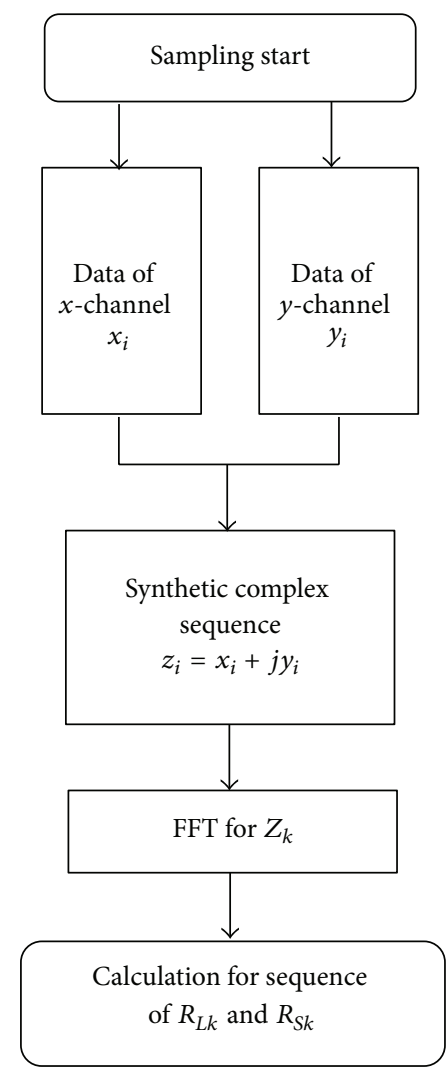

Figure 7: Full-vector fusion calculation.

However, after observing the signal in the $y$-direction, it seems that the vibration relates to the rotating frequency; the fault may be related to the unbalance. Different fault conclusions may be drawn on the machine by different signals. The synthetic signal of $x$ and $y$ transducer at each harmonic is a motion ellipse. The major axis of the ellipse reflects the most significant vibration. No matter where the transducer mounts, the main vibration vector is the maximum amplitude of the vibration in the motion plane. On the same rotor section, the main vibration vector is not affected by the radial mounting position of the two transducers installed vertically with each other. The full-vector spectrum is the synthesis of two homologous transducers' information [18]. It works as a virtual transducer in the plane to detect the maximum vibration of the interface at each harmonic. So its spectral structure can reflect the operation state of the machine comprehensively.

$R_{L k}$ is half major axis of the ellipse at the frequency point $k$. The spectrum of $R_{L k}$, called full-vector spectrum, is the synthesis of two homologous transducers' information and

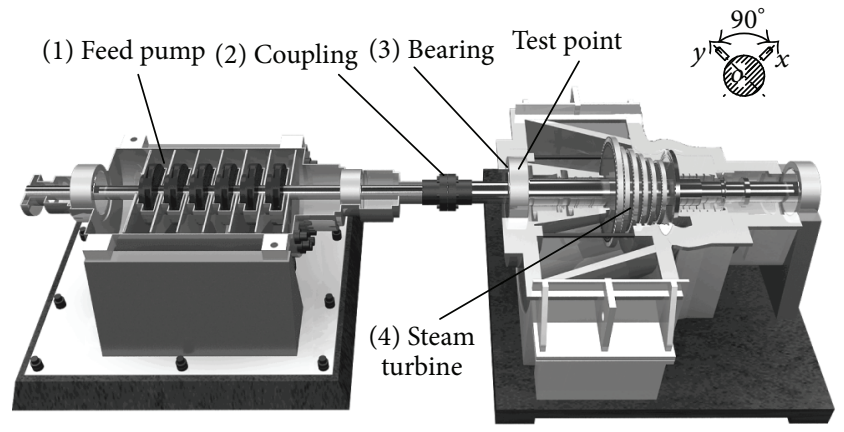

FIGURE 8: Machine structure and point position.

is not affected by the radial mounting position of the two transducers installed vertically with each other. Comparing with the spectrum in one direction only, its spectral structure can reflect the operation state of the machine comprehensively. Autoregressive (AR) model can be used for time series vibration analysis. Figure 10 is a series of predicted full-vector spectrums based on time series signals. The order of the AR model is 13 . The signals were acquired from a steam turbine. High pressure rotor speed of the turbine is $3000 \mathrm{r} / \mathrm{min}$; sampling frequency is $1600 \mathrm{~Hz}$; sampling points are 1024 . The AR model was constructed by 67 sets of vibration data. Since change trend of vibration amplitude of each harmonic is different, the AR models were constructed for the time series of each harmonic. The spectrum predicted model is an AR model array. Since the fault characteristic is concerned with the spectrum structure, the characteristic and position of machine fault which may occur in the future can be determined by analysis of the predicted full-vector spectrum. By this means, one may now determine a potential fault such as cracked rotor, bad bearing, and seal instability and make a reasonable arrangement of equipment maintenance.

4.2. Discussion. The main vector sequence $R_{L k}(k=$ $0,1,2, \ldots, N / 2-1)$ reflects the distribution of the vibration energy along the frequency. Comparing with the spectrum in one direction only, its spectral structure can reflect the operation state of the machine comprehensively. At the same test position, spectrum structure with one installation direction may be different from that of another direction. On the same rotor section, the full-vector spectra's structure is not affected by the radial mounting position of the two transducers installed vertically with each other. The full-vector spectrum is the true reflection of the vibration characteristics. The characteristic and position of machine fault which may occur in the future can be determined by analysis of the predicted full-vector spectrum. 


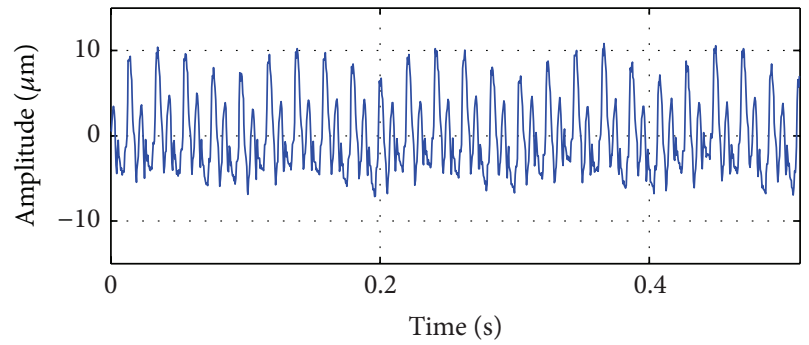

(a)

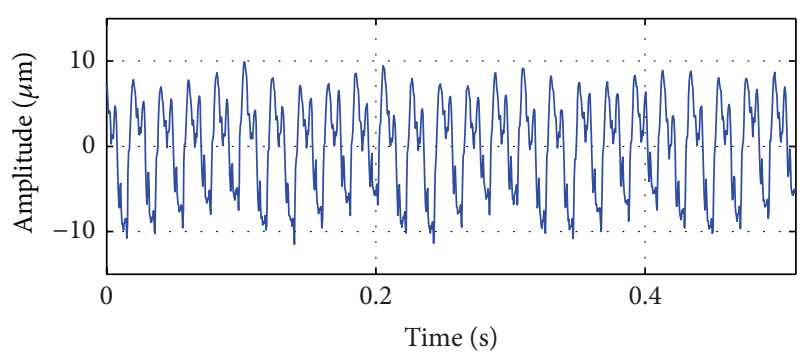

(c)

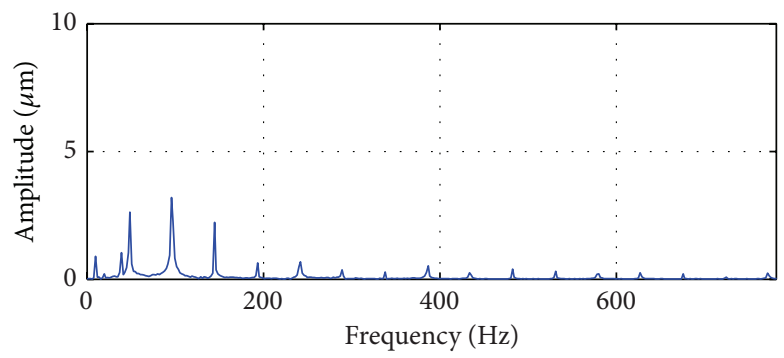

(b)

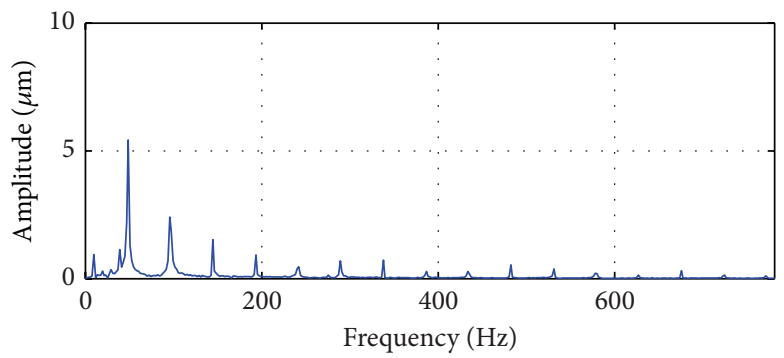

(d)

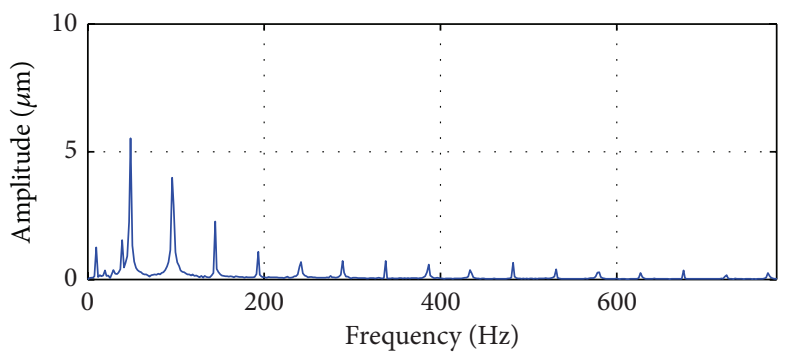

(e)

FIGURE 9: (a) Vibration wave of $x$-direction; (b) spectrum of $x$-direction; (c) vibration wave of $y$-direction; (d) spectrum of $y$-direction; (e) full-vector spectrum.

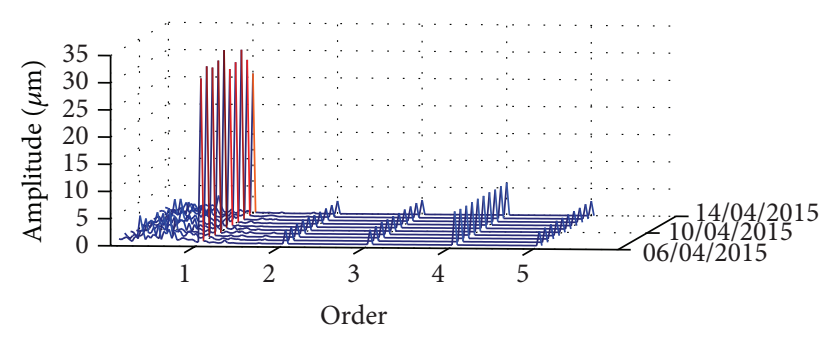

FIGURE 10: Results of full-vector spectrum prediction.

\section{Conclusions}

The machine running condition can be predicted by means of fault prediction. Usually, the fault prediction uses the datadriven model. Traditionally, the signals are acquired by one or more transducers. Industry wide of a single transducer to assess dynamics has been replaced by use of two or more transducers and key-phasor as fully described in detail in the classic works by Dr. Bently et al. [18]. However, researches on mechanical fault prediction, currently, are still mainly focused on the forecasting of single specific fault trend and evaluating equipment's residual life. In this study, the application has been carried out on the basis of the methodologies proposed by Dr. Bently and Dr. Muszynska. The spectrum based on one transducer has disadvantages of uncertainty and one-sidedness. The proposed full-vector data acquisition and information fusion method for fault prediction effectively overcame the above problems. In theory, the data acquisition system, on the one hand, can eliminate the uncertainty of the spectrum structure caused by a single vibration signal; on the other hand, data fusion in the data acquisition layer can also provide a feasible method for the establishment of the full-vector prediction model. The study on full-vector data acquisition system lays the technical foundation for the further research of the prediction and diagnosis of fault characters.

\section{Competing Interests}

The authors declare that there is no conflict of interests regarding the publication of this paper. 


\section{Acknowledgments}

This project is supported by the Guidance Program of the Key Projects of Scientific and Technical Research of Department of Education of Henan Province (13B603970.0), Natural Science Research Program of Department of Education of Henan Province (2010B460015), and the Opening Project of Key Laboratory of Precision Manufacturing Technology and Engineering, Henan Polytechnic University (PMTE201302A).

\section{References}

[1] Z. Y. Xue, T. J. Yang, and J. H. Zhu, "Review of medels in machinery fault prediction," Journal of Mechanical Strength, S1, pp. 60-65, 2006.

[2] L. Yong-Kuo, P. Min-Jun, X. Chun-Li, and D. Ya-Xin, "Research and design of distributed fault diagnosis system in nuclear power plant," Progress in Nuclear Energy, vol. 68, pp. 97-110, 2013.

[3] Z. Liu, Y. Liu, D. Zhang, B. Cai, and C. Zheng, "Fault diagnosis for a solar assisted heat pump system under incomplete data and expert knowledge," Energy, vol. 87, pp. 41-48, 2015.

[4] L. Lin and F. L. Chu, "HHT-based AE characteristics of natural fatigue cracks in rotating shafts," Mechanical Systems and Signal Processing, vol. 26, no. 1, pp. 181-189, 2012.

[5] C. M. Stoisser and S. Audebert, "A comprehensive theoretical, numerical and experimental approach for crack detection in power plant rotating machinery," Mechanical Systems and Signal Processing, vol. 22, no. 4, pp. 818-844, 2008.

[6] A. A. Gubran and J. K. Sinha, "Shaft instantaneous angular speed for blade vibration in rotating machine," Mechanical Systems and Signal Processing, vol. 44, no. 1-2, pp. 47-59, 2014.

[7] S. Hou and Y. R. Li, "Short-term fault prediction based on support vector machines with parameter optimization by evolution strategy," Expert Systems with Applications, vol. 36, no. 10, pp. 12383-12391, 2009.

[8] Q. X. Zhu, Y. W. Jia, D. Peng, and Y. Xu, "Study and application of fault prediction methods with improved reservoir neural networks," Chinese Journal of Chemical Engineering, vol. 22, no. 7, pp. 812-819, 2014.

[9] A. Kusiak and W. Li, "The prediction and diagnosis of wind turbine faults," Renewable Energy, vol. 36, no. 1, pp. 16-23, 2011.

[10] J. K. Sinha and K. Elbhbah, "A future possibility of vibration based condition monitoring of rotating machines," Mechanical Systems and Signal Processing, vol. 34, no. 1-2, pp. 231-240, 2013.

[11] X. L. Zhang, W. Chen, B. J. Wang, and X. F. Chen, "Intelligent fault diagnosis of rotating machinery using support vector machine with ant colony algorithm for synchronous feature selection and parameter optimization," Neurocomputing, vol. 167, pp. 260-279, 2015.

[12] F. Serdio, E. Lughofer, K. Pichler, T. Buchegger, M. Pichler, and H. Efendic, "Fault detection in multi-sensor networks based on multivariate time-series models and orthogonal transformations," Information Fusion, vol. 20, no. 1, pp. 272-291, 2014.

[13] M. S. Safizadeh and S. K. Latifi, "Using multi-sensor data fusion for vibration fault diagnosis of rolling element bearings by accelerometer and load cell," Information Fusion, vol. 18, no. 1, pp. 1-8, 2014.

[14] P. Boškoski, M. Gašperin, and D. Petelin, "Bearing fault prognostics based on signal complexity and Gaussian process models," in Proceedings of the IEEE Conference on Prognostics and Health Management (PHM '12), pp. 1-8, Denver, Colo, USA, June 2012.

[15] P. Boškoski, M. Gašperin, D. Petelin, and D. Juričić, "Bearing fault prognostics using Rényi entropy based features and Gaussian process models," Mechanical Systems and Signal Processing, vol. 52-53, no. 1, pp. 327-337, 2015.

[16] J. Han and L. D. Shi, Full Vector Spectrum Technology and Its Engineering Application, China Machine Press, Beijing, China, 2008.

[17] Y. Y. Li, J. Han, L. Chen et al., "Research on condition trend prediction method of mechanical equipment based on ARKalman," Manufacturing Automation, no. 12, pp. 95-96, 120, 2014.

[18] D. E. Bently, C. T. Hatch, and B. Grissom, Fundamentals of Rotating Machinery Diagnostics, Bently Pressurized Bearing Press, Minden, Nev, USA, 2002.

[19] A. Muszynska, Rotordynamics, Taylor \& Francis Group, New York, NY, USA, 2005.

[20] M. L. Adams, Rotating Machinery Vibration: From Analysis to Troubleshooting, Marcel Dekker, New York, NY, USA, 2001.

[21] C. Chattoraj, S. N. Sengupta, and M. C. Majumder, "Dynamics of a verticalJeffcott rotor, Bull Eng Sci, vol. 3, no. 2, pp. 1-10, 2008.

[22] J. M. Vance, Rotordynamics of Turbomachinery, WileyInterscience, New York, NY, USA, 1987.

[23] L. Chen, J. Han, W. P. Lei et al., "Vibration phase measurement method based on embedded system," Chinese Journal of Construction Machinery, vol. 13, pp. 153-157, 2015. 




\section{Enfincering}
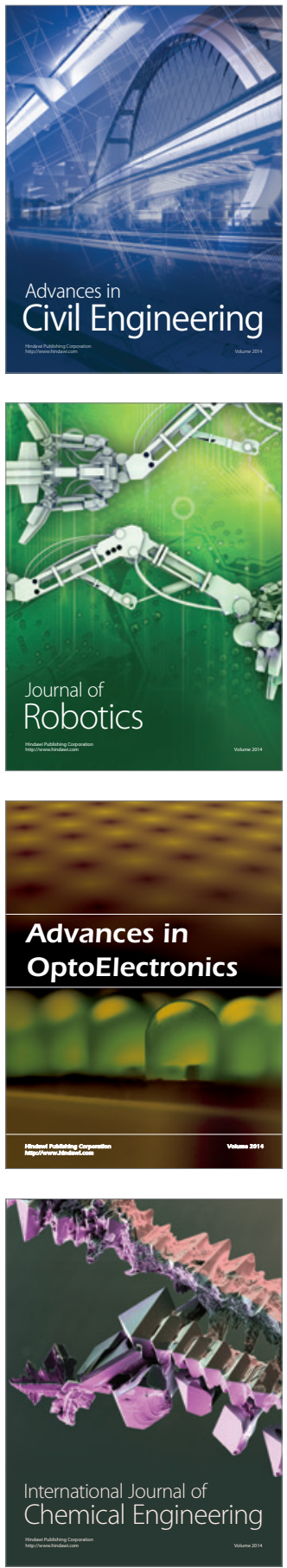

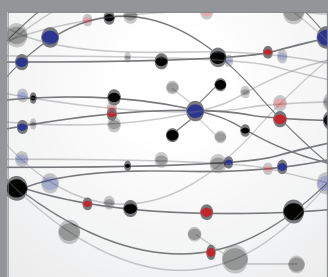

The Scientific World Journal

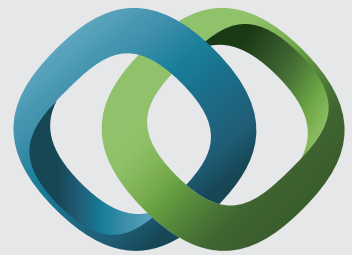

\section{Hindawi}

Submit your manuscripts at

http://www.hindawi.com
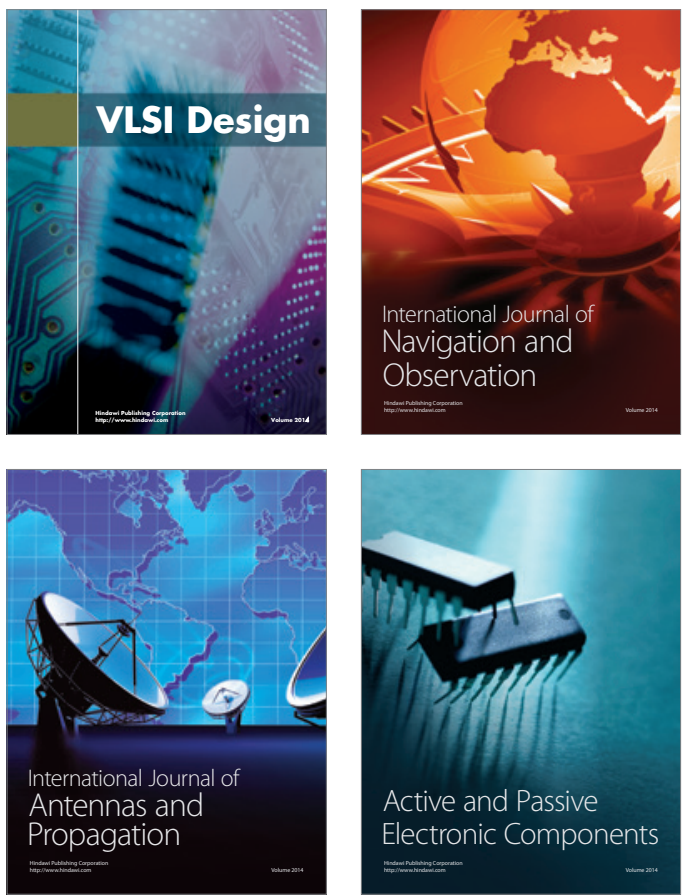
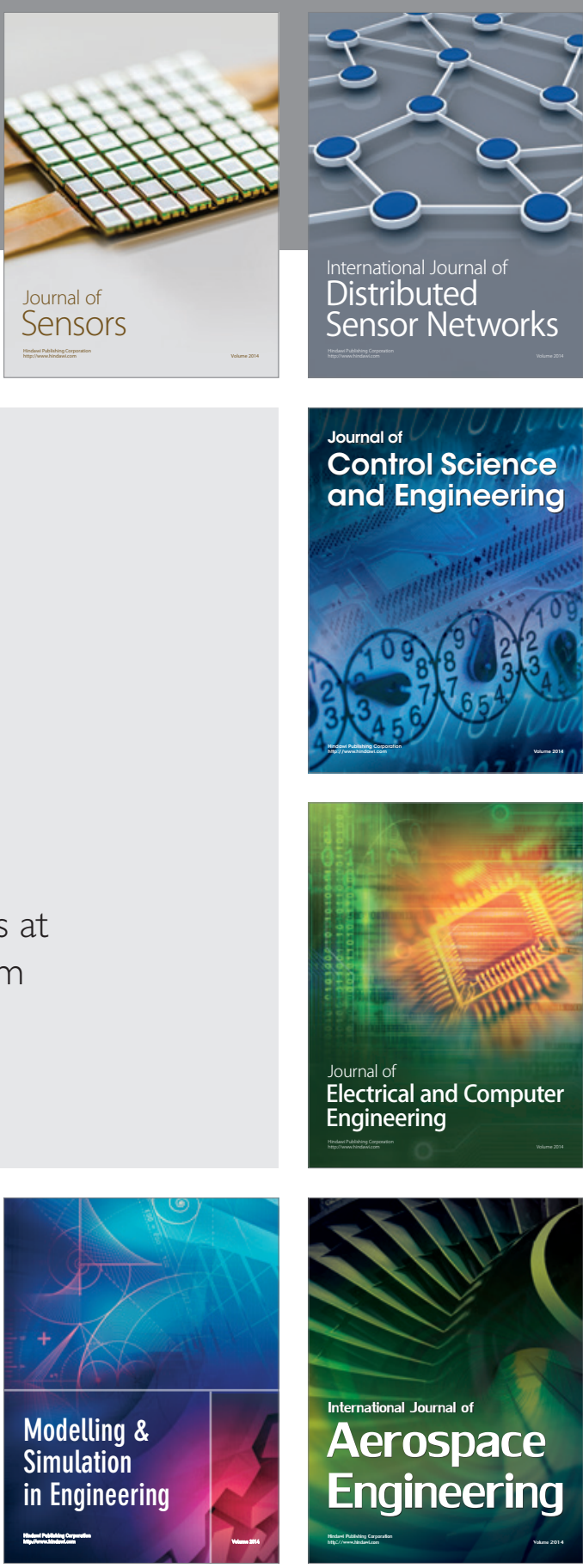

International Journal of

Distributed

Sensor Networks

Journal of

Control Science

and Engineering
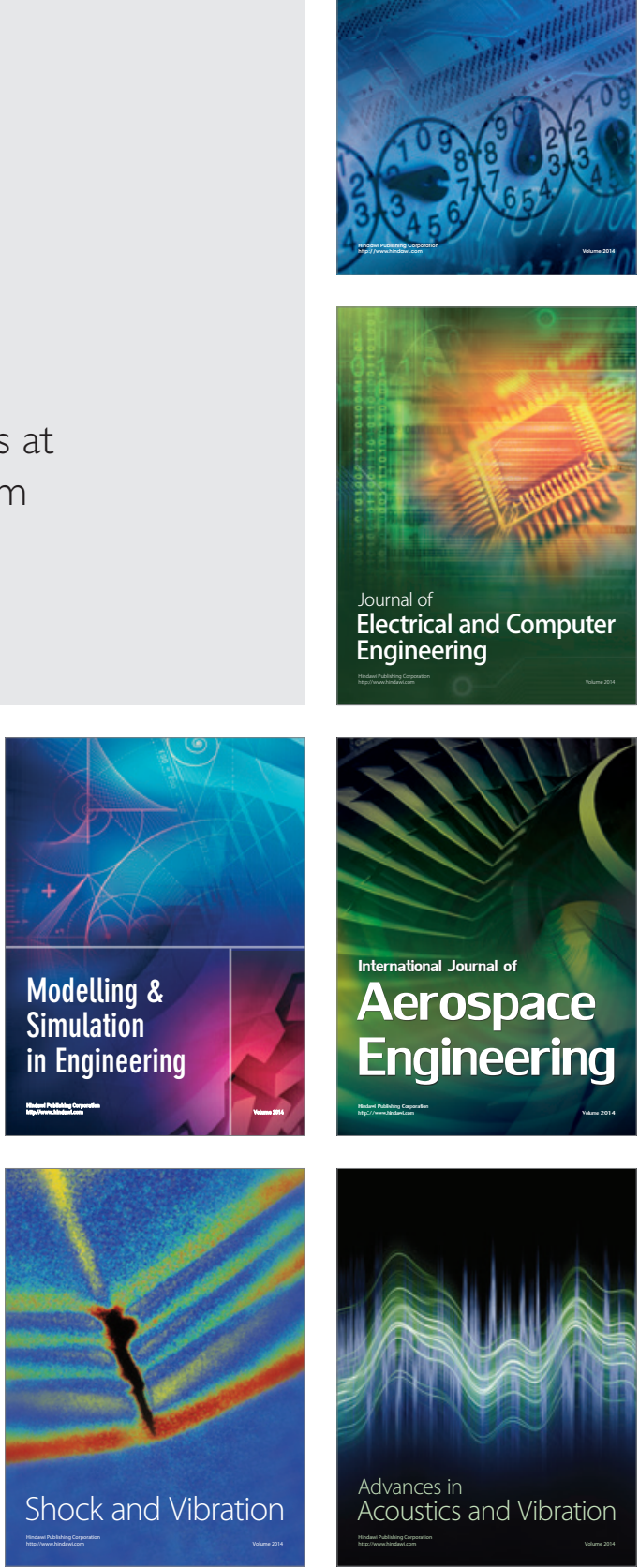